\title{
Five-Millimeter Port Site Hernia: A Rare Case
}

\author{
Daria Abolghasemi ${ }^{\mathrm{a}}$, Julia Arena ${ }^{\mathrm{b}, \mathrm{d}}$, Andrew Camerota ${ }^{\mathrm{c}}$
}

\begin{abstract}
With advanced technology and improved training of surgeons, laparoscopic and other minimally invasive surgical techniques have become the standard of treatment for many general surgical and gynecological diseases. As with any surgical procedure, there are complications that can be directly attributed to the surgical technique. We present a case of an 84-year-old female who presented with symptoms of small bowel obstruction secondary to a 5-mm port site hernia (PSH) 1 week post-laparoscopic bilateral salpingo-oophorectomy for ovarian cysts.
\end{abstract}

Keywords: Port site hernia; Trocar site hernia; 5-mm

\section{Introduction}

Minimally invasive surgical techniques, primarily by means of laparoscopic visualization, began to come into favor most abundantly within the field of gynecological surgery within the 1970's and 1980's [1]. The advent of newly developed laparoscopic equipment allowed for the treatment of conditions including endometriosis, tubal pregnancies, and ovarian masses with a reduction in post-operative pain and improvement in recovery period. This technique was adapted into standard general surgical practice prominently with its initial usage in cholecystectomy. Similarly, such procedures resulted in decreased post-operative pain, shortened hospitalization, and early return to normal pre-operative level of functioning. Laparoscopic-assisted procedures continued to progress the manner in which general surgeries were performed, expanding their use to colectomies as well as single-incision, endoscopic, and robotic operations.

Advanced surgical technique is not without perioperative complications. Port site hernia (PSH) is a prime example of this, and is of particular interest given the requirement for

Manuscript submitted July 7, 2018, accepted July 20, 2018

aSt. Joseph's Regional Medical Center, 703 Main Street, Paterson, NJ 07503, USA

bNew York Medical College, 40 Sunshine Cottage Road, Valhalla, NY 10595, USA

'Department of Surgery, St. Peter's University Hospital, 254 Easton Avenue, New Brunswick, NJ 08901, USA

${ }^{\mathrm{d} C}$ Corresponding Author: Julia Arena, New York Medical College, 40 Sunshine Cottage Road, Valhalla, NY 10595, USA. Email: Juarena89@gmail.com

doi: https://doi.org/10.14740/jcs353w surgical correction. With increased propensity for minimally invasive surgery, the incidence of this complication and subsequent prevention is continuously addressed. Particularly, there is a need to evaluate PSH at $5-\mathrm{mm}$ incision sites given their rarity, with literature reporting an incidence of $0.65-2.8 \%$ [2]. We present a case of the repair of a 5-mm PSH following a laparoscopic bilateral salpingo-oophorectomy.

\section{Case Report}

An 84-year-old female with a body mass index (BMI) of $32.42 \mathrm{~kg} / \mathrm{m}^{2}$ and a past medical history of hypertension, dyslipidemia, hypothyroidism and glaucoma presented to the emergency department (ED) with complaints of vomiting and poor oral intake for approximately 1 week. Seven days prior to arrival, the patient had undergone a laparoscopic bilateral salpingo-oophorectomy for ovarian cysts. Post-operatively, she stated that she was passing flatus, but had only one bowel movement since her discharge. She also reported vomiting daily and had oral intake intolerance since her procedure. At the time of presentation to the ED the patient was in no acute distress and her vitals were as follows: temperature $36.4{ }^{\circ} \mathrm{C}$, heart rate 82 beats per minute, and blood pressure $154 / 82 \mathrm{~mm}$ $\mathrm{Hg}$. Her abdominal exam was significant for mild abdominal distention, but no tenderness or guarding was elicited. Her surgical wounds were clean and intact, and she was noted to have a mass-like fullness over her right lower quadrant $5-\mathrm{mm}$ port site incision. Computed tomography (CT) scan and plain film X-ray performed in the ED demonstrated findings suggestive of a small bowel obstruction caused by a herniated loop of small bowel in the right lower quadrant, consistent with the location of the laparoscopic port site (Fig. 1). A complete blood count and basic metabolic panel were within normal limits. She was admitted to the surgical service, made nil per os (NPO), given IV fluids, had a nasogastric tube placed for decompression and she was scheduled for surgical repair. After admission, the patient underwent an open right-sided incisional hernia repair. A small right lower quadrant transverse incision was made over the hernia site. A portion of small bowel with omentum was found to be incarcerated within the defect. After careful inspection, this portion of the bowel was found to be viable and was reduced into the abdominal cavity. The defect was repaired primarily with a \#0 Prolene suture. Post-operatively, the patient returned to the surgical floor for monitoring. On post-operative day 1, her diet was advanced as tolerated, she began passing flatus and was having normal bowel movements. She was subsequently discharged to home on post-operative day 2 without any further post-operative 

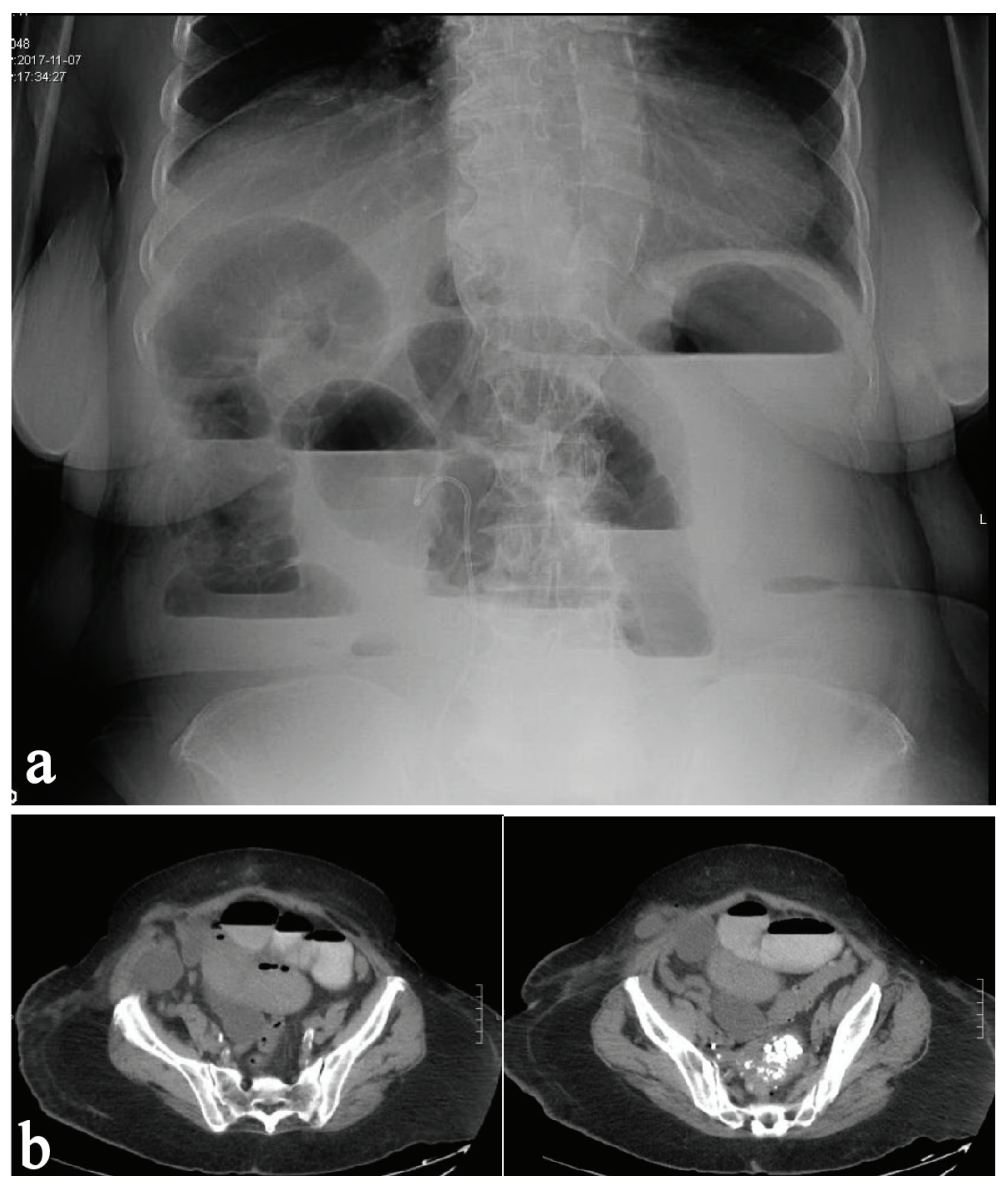

Figure 1. (a) Plain film X-ray of patient's abdomen at ED presentation demonstrating air-fluid levels. (b) Computed tomography scan of patient's abdomen at ED presentation demonstrating a herniated loop of bowel with air-fluid levels.

events or complications.

\section{Discussion}

PSH is defined as the development of herniation of intra-abdominal contents at a trocar site. The development of PSH following laparoscopic procedures is a well-known complication of minimally invasive surgeries, the first of which was reported in 1968 [3]. Since this time, efforts have focused on assessing the incidence of PSH, risk factors for their development, preventive measures, and how best to address the complication when PSH do occur. Given the very low incidence of PSH at 5-mm trocar sites, literature on this topic is limited.

Patient risk factors for the development of PSH include age greater than 60 years, male sex, obesity, parity, pre-existing fascial defect, surgical site infection and poor healing secondary to diabetes/steroid use/poor nutritional status [4]. With regards to parity, it is believed that parous women have abdominal wall and fascial laxity resulting in tissue that is vulnerable to developing fascial defects. Operative risk factors include trocar diameter, prolonged operative time and in turn prolonged manipulation of port sites, and an observed increased incidence of PSH with the use of bladed/cutting trocars in comparison to blunted (conical, pyramidal, radially dilating, non-bladed) trocars [5]. Additionally, it has been postulated that the creation of a pneumoperitoneum may result in omentum or intestinal loop herniation through the point of insertion in the fascia following retrieval of trocars. For this reason, adequate deflation of the pneumoperitoneum prior to trocar extraction is encouraged to reduce this complication.

In high risk operations, consideration should be given to closure of all insertion sites. Tonouchi et al reported that primary closure of fascial defects resulted in fewer PSH than when defects were left open. However, many surgeons opt to leave fascia unclosed to avoid injury of underlying bowel loops, omentum or abdominal organs as closure can be a challenging task, especially concerning with high risk procedures [6]. Consideration of primary fascial closure should be made by the operating surgeon if extensive manipulation has occurred (i.e. multiple sheath reinsertions, organ retrieval, prolonged operative time), or if there are pre-existing patient risk factors (obesity, pre-existing fascial defects, poor nutritional status). There is no reported benefit of one closure technique over another method (CT-NP device vs. Endoclose vs. standard hand-sutured closure) [7], and therefore this decision is based on operator preference. Given that there is inadequate conclusive data reporting proper guidelines for closure of 5-mm port 
sites, the decision of when to do so is left to the discretion of the operating surgeon.

\section{References}

1. Lee-Kong S, Feingold D. The history of minimally invasive surgery. Semin Colon Rectal Surg. 2013;24:3-6.

2. Tonouchi H, Ohmori Y, Kobayashi M, Kusunoki M. Trocar site hernia. Arch Surg. 2004;139(11):1248-1256.

3. Fear RE. Laparoscopy: a valuable aid in gynecologic diagnosis. Obstet Gynecol. 1968;31(3):297-309.

4. Pereira N, Hutchinson AP, Irani M, Chung ER, Lekovich
JP, Chung PH, Zarnegar R, et al. 5-millimeter trocar-site hernias after laparoscopy requiring surgical repair. J Minim Invasive Gynecol. 2016;23(4):505-511.

5. Huang B, Seow K, Tsui K, Su W, Lu C, Wang P. Small trocar site hernia after laparoscopy. Gynecology and Minimally Invasive Therapy. 2013;2(3):79-84.

6. Ece I, Yilmaz H, Alptekin H, Yormaz S, Colak B, Sahin M. Port site hernia after laparoscopic sleeve gastrectomy: a retrospective cohort study of 352 patients. Updates Surg. 2018;70(1):91-95.

7. Yamamoto M, Minikel L, Zaritsky E. Laparoscopic 5-mm trocar site herniation and literature review. JSLS. 2011;15(1):122-126. 